\section{Commentary: Planning ahead: Fetal magnetic resonance imaging may predict brain injury before surgery for congenital heart disease}

\author{
Mohan J. John, MD, and Travis J. Wilder, MD
}

Despite excellent survival following surgery for congenital heart disease (CHD) in the modern era, children with CHD have worse long-term neurodevelopmental and functional outcomes compared with their peers. ${ }^{1}$ In an effort to improve neurodevelopmental outcomes, recent research efforts have focused on identifying the mechanisms and patterns of neurologic injury in this population. Initially, and somewhat intuitively, it was assumed that brain injury occurred as a consequence of cardiopulmonary bypass and deep hypothermic circulatory arrest or antegrade cerebral perfusion. However, advanced imaging shows that brain abnormalities occur in neonates with CHD even before cardiac intervention. ${ }^{2}$ This suggests that brain injury and neurodevelopmental outcomes are related to the cumulative effect of patient physiology, along with preoperative, operative, and postoperative risk factors (Figure 1). ${ }^{1}$

For patients with CHD, the predominant pattern of brain injury is diffuse or focal white matter injury (WMI). Interestingly, similar findings are seen on brain imaging in preterm newborns. ${ }^{3}$ White matter maturation is delayed in both populations, which increases susceptibility to injury. Patients with complex CHD have decreased fetal delivery of oxygen and nutrients to the developing brain, leading to impaired brain growth and dysmaturity. The cellular

\footnotetext{
From the Division of Congenital Heart Surgery, Texas Children's Hospital, Baylor College of Medicine, Houston, Tex.

Disclosures: The authors reported no conflicts of interest.

The Journal policy requires editors and reviewers to disclose conflicts of interest and to decline handling or reviewing manuscripts for which they may have a conflict of interest. The editors and reviewers of this article have no conflicts of interest.

Received for publication Nov 25, 2020; revisions received Nov 25, 2020; accepted for publication Nov 30, 2020; available ahead of print Dec 14, 2020.

Address for reprints: Travis J. Wilder, MD, Legacy Tower, Floor 19, 6651 Main St, Mail Code 19345H, Houston, TX 77030 (E-mail: tjwilder@texaschildrens.org). J Thorac Cardiovasc Surg 2021;162:1015-6 $0022-5223 / \$ 36.00$

Copyright (c) 2020 by The American Association for Thoracic Surgery https://doi.org/10.1016/j.jtcvs.2020.11.141
}

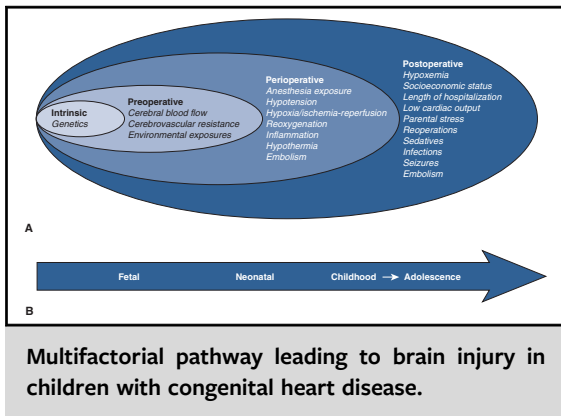

CENTRAL MESSAGE

Preoperative differences in physiology associated with various congenital heart defects may be an important risk factors for brain injury and subsequent neurodevelopment.

mechanisms responsible for CHD-induced brain injury, however, are not well elucidated.

Peyvandi and colleagues ${ }^{4}$ sought to better understand the relationship between brain immaturity and WMI among patients with hypoplastic left heart syndrome (HLHS) and transposition of the great arteries (TGA). They performed third-trimester and preoperative (postnatal) brain magnetic resonance imaging (MRI) on 63 subjects. Total brain volume (TBV) was used as a measure of brain maturity, and moderate-to severe WMI was the outcome of interest. Although WMI was not identified on fetal MRI in any of the patients, about one third of patients in both groups had evidence of WMI on preoperative MRI. Fetal brain growth did not differ between the groups. TBV was significantly lower in patients with moderate-to-severe WMI and TGA but not in patients with HLHS. Among patients with TGA, ventricular septal defect did not alter the incidence of significant WMI. Patients with HLHS and aortic atresia had a greater incidence and severity of WMI.

Taken in aggregate, these findings suggest that the mechanism of brain injury may differ based on fetal physiology-specifically, between patients with TGA and HLHS. TBV may be helpful to predict the susceptibility of the brain to injury for patients with TGA but not for patients with HLHS. The clinical implication is that newborns with TGA found to have smaller brain volumes on fetal MRI may benefit from targeted strategies to increase cerebral oxygen delivery, potentially reducing the incidence of WMI. 


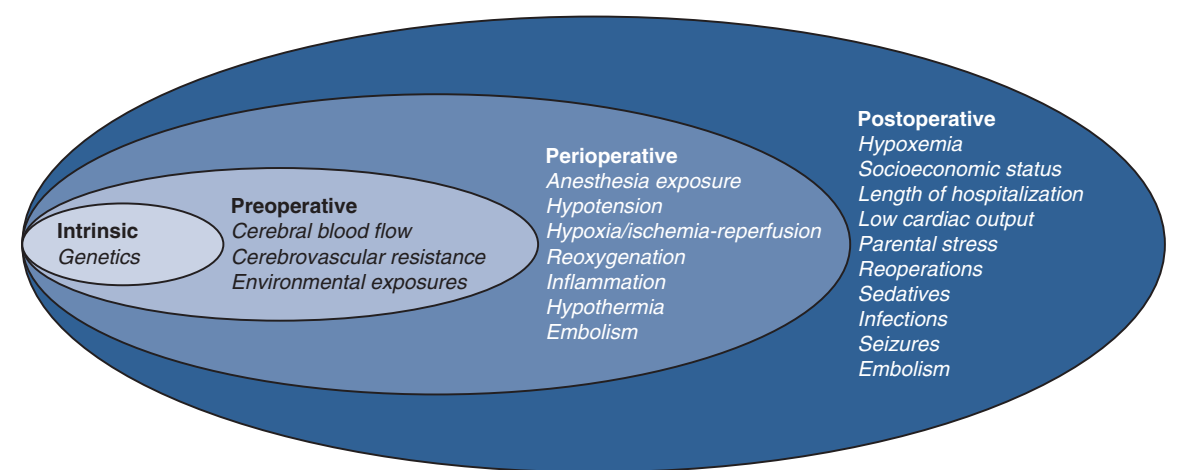

A

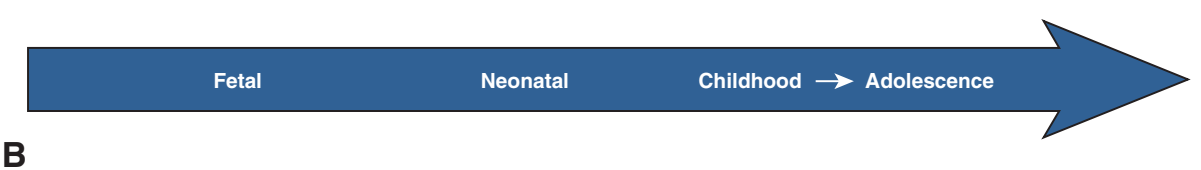

FIGURE 1. Multifactorial pathway leading to brain injury in children with congenital heart disease.

Understandably, the study by Peyvandi and colleagues is limited by small numbers in each subset of patients, leading to a lack of granular detail. In addition, their assessment of brain maturity was restricted to brain volumes, without mention of additional supportive findings related to myelination, cortical folding, germinal matrix distribution, and glial cell migration. ${ }^{5}$ Despite these limitations, this study provides original data to help expand our understanding of the patterns of brain development and injury in patients with complex CHD. Moreover, longitudinal follow-up will likely add valuable insight on the long-term neurodevelopmental progress among these patients.

\section{References}

1. Morton PD, Ishibashi N, Jonas RA. Neurodevelopmental abnormalities and congenital heart disease: insights into altered brain maturation. Circ Res. 2017; 120:960-77.

2. Donofrio MT, Duplessis AJ, Limperopoulos C. Impact of congenital heart disease on fetal brain development and injury. Curr Opin Pediatr. 2011;23: $502-11$.

3. Miller SP, McQuillen PS. Neurology of congenital heart disease: insight from brain imaging. Arch Dis Child Fetal Neonatal Ed. 2007;92:F435-7.

4. Peyvandi S, Lim JM, Marini D, Xu D, Reddy VM, Barkovich AJ, et al. Fetal brain growth and risk of postnatal white matter injury in critical congenital heart disease. J Thorac Cardiovasc Surg. 2021;162:1007-14.e1.

5. Childs AM, Ramenghi LA, Cornette L, Tanner SF, Arthur RJ, Martinez D, et al. Cerebral maturation in premature infants: quantitative assessment using MR imaging. AJNR Am J Neuroradiol. 2001;22:1577-82. 